\title{
Fuel retention over a full day of experiments in JET
}

\author{
T Loarer ${ }^{1}$, J Bucalossi ${ }^{1}$, D Brennan², G Matthews², Ph Morgan², V Philipps ${ }^{3}$, \\ M Stamp $^{2}$, E Tsitrone ${ }^{1}$ and JET EFDA contributors \\ ${ }^{1}$ Association EURATOM-CEA, DSM-DRFC, CEA Cadarache, \\ 13108 St Paul lez Durance, France. \\ ${ }^{2}$ Euratom-UKAEA Fusion Association, Culham Science Centre, Abingdon, OX14 3EA, \\ UK. \\ 3IPP, Forschungszentrum Juelich, D-52425 Juelich, Germany
}

\section{I - Introduction}

Particle control is a crucial point for the next step machines : particle injection and extraction systems must regulate the D-T fuel densities, exhaust helium ash, control the impurity core contamination and minimize the tritium wall inventory [1]. Most of our knowledge on long-term fuel retention is from post mortem surface analysis of tiles, which will, however, not be possible in ITER. Thus the evaluation of gas balance data in present tokamaks is of high priority to establish the technique and database for ITER, in which gas balance will very likely be the dominant technique to assess the fuel retention. A series of identical discharges has been performed in JET for a full day, in order to study the particle retention behaviour as a function of the wall inventory and the global balance for a significant number of discharges associated to a high gas injection. The resulting particle balance over this day is presented in this paper.

\section{II - Experiments}

A series of 39 consecutive and repetitive $L$ mode discharges has been performed in JET with the following main parameters: $\mathrm{I}_{\mathrm{p}}=2 \mathrm{MA}, \mathrm{B}_{\mathrm{T}}=2.4 \mathrm{~T},<\mathrm{n}_{\mathrm{e}}>=3.810^{19} \mathrm{~m}^{-3}$, gas rate 1.5

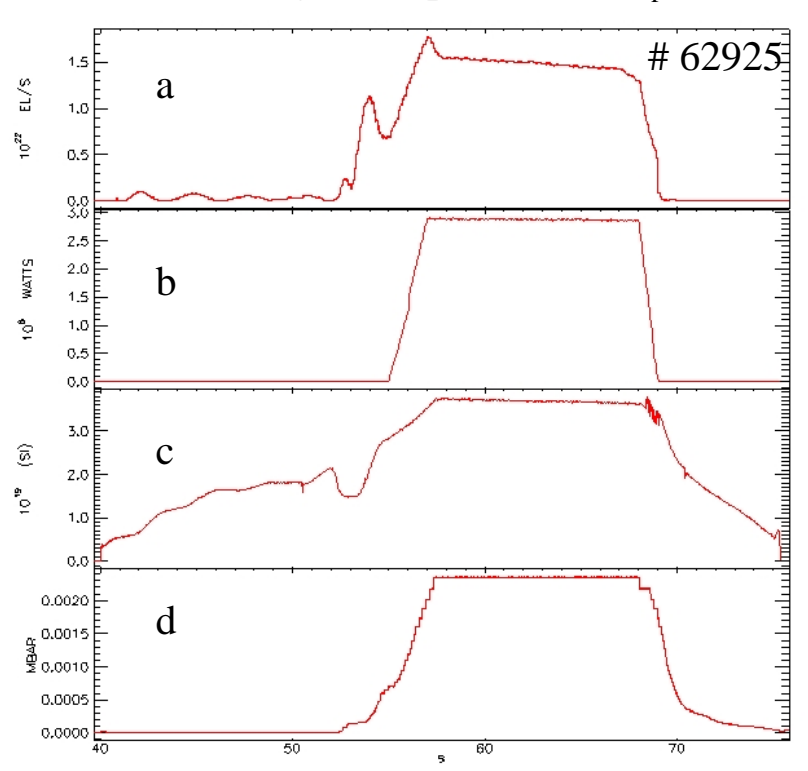

Figure 1: Time evolution of the gas injection (a), ICRH heating (b), plasma density (c) and neutral pressure(d) in the $10^{22} \mathrm{Ds}^{-1}$ injected from the top of the machine and with $2.8 \mathrm{MW}$ of ICRH for auxiliary heating over a duration of 11s. The plasma geometry in the divertor is a "DOCL” (Diagnostic Optimized Configuration) configuration, the inner and outer legs are located on the vertical target at about $10 \mathrm{~cm}$ for the bottom of the targets. Figure 1 displays the time evolution of the main plasma parameters, showing steady- state conditions over the 11 sec of auxiliary heating on every shot. The cumulative gas injected is $15901 \mathrm{Pam}^{3}$ of $\mathrm{D}_{2}\left(7.85510^{24} \mathrm{D} @\right.$ $20^{\circ} \mathrm{C}$ ) for a total plasma duration of 1400 sec (corresponding to 3.5 ITER discharges but with lower gas injection rate). Since the active pumping was achieved using the cryopump only (the valves of the beam boxes and of the 
turbo pumps were closed), its regeneration has allowed us to calibrate the value of the pumped particle flux (Pumping speed of the cryopump associated with a neutral pressure measurement by a pressure gauge located $\sim 2.5 \mathrm{~m}$ below the cryopump) to be used in the particle balance analysis during plasma operations.

The global balance over the full day requires also a precise knowledge of the gas released between the pulses. The resulting pumping speed of the cryopump in the vessel is estimated from the "dry runs" carried out at the beginning of the day. In the absence of plasma and with the cryopump only, the total number of particles pumped is equal to the total number of particles injected : $\mathrm{Q}_{\text {injected }}=S_{\text {cryo }}^{\text {ves }} \int \mathrm{P}_{\text {vessel. }} \mathrm{dt}$, where $\mathrm{P}_{\text {vessel }}$ is the neutral pressure measured in the equatorial plane of the vessel. In this estimation, the natural gas release is neglected (and is negligible) over the dry run duration $(<100 \mathrm{~s})$. This allows us to determine the resulting pumping speed of the cryopump in the vessel in the absence of plasma : $S_{\text {cryo }}^{\text {ves }}=66 T_{\text {gauge }}^{\text {vessel }} / 273.15 \mathrm{~m}^{3} \mathrm{~s}^{-1}$ where $T_{\text {gauge }}^{\text {vesel }}$ is the temperature of the gas whose pressure is measured by the vessel gauge.

Using this value for the full day, the total gas released between the discharges is 2385 $\operatorname{Pam}^{3}\left(1.17810^{24} \mathrm{D}\right)$. Since the overall gas balance for the full day exhibits an equilibrium between the total injected and the total regenerated, this allows us to deduce the corresponding pumping speed of the cryopump during plasma operation, with reference to the pressure gauge located below the divertor pump. This pumping speed can be expressed as $S_{\text {cryo }}^{\text {ves }}=115 T_{\text {gauge }}^{\text {divetor }} / 273.15 \mathrm{~m}^{3} \mathrm{~s}^{-1}$ where $\mathrm{T}_{\text {gauge }}^{\text {divertor }}$, is the temperature of the gas

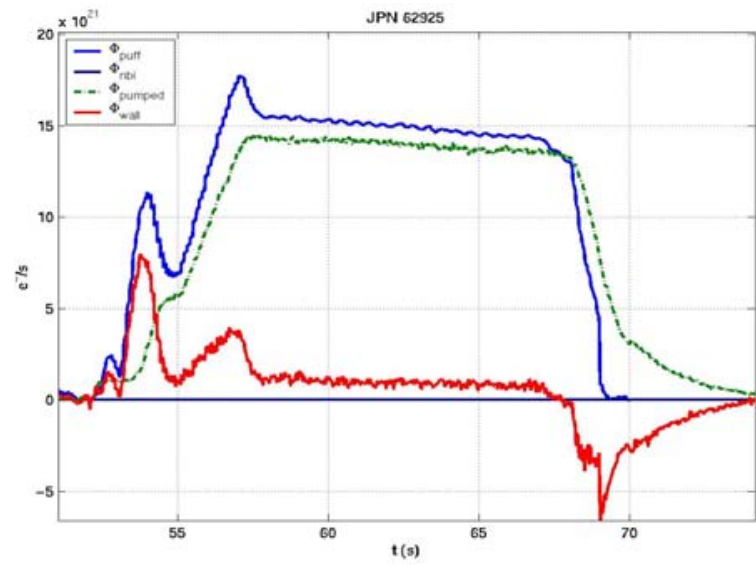

Figure 2: Time evolution of particle fluxes $\Gamma_{\text {puff, }} \Gamma_{\text {exhauted }}$, and resulting wall particle retention $\Gamma_{\text {wall }}$. whose pressure is measured by the pressure gauge located below the divertor.

The cumulative particles exhausted by the cryopump during the plasma experiments is $13516 \mathrm{Pam}^{3}\left(6.67710^{24} \mathrm{D}\right)$ while the vessel inventory is $2385 \mathrm{Pam}^{3}$, which represents less than $6 \%$ of the particles injected and released between pulses. In figure 2, the time evolution of the particle fluxes, gas injection, pumped flux and resulting wall loading are plotted as a function of time for a characteristic pulse of the series. Except at the beginning of the injection ( $\sim 3$ to $58 \mathrm{sec}$ ), the wall loading is shown to be constant as a function of time; the retention defined as Ret $=\left(\Gamma_{\text {inj }}-\Gamma_{\text {exh }}\right) / \Gamma_{\text {inj }}$, is less than $8 \%$ during the $11 \mathrm{sec}$ of the ICRH power [57-68 sec]. At the end of the plasma, 5.3\% of the particles injected are retained in the machine which are recovered before the next discharge about 20 minutes later. For the series of experiments, the recovery from pulse to pulse is shown to be very reproducible.

\section{III - Effect of the plasma geometry}

For given plasma parameters, the resulting neutral pressure in the subdivertor is shown to be very sensitive to the location of the inner and outer strike points. Figure 3 displays the time evolution of a pulse with $\mathrm{I}_{\mathrm{p}}=2 \mathrm{MA}, \mathrm{B}_{\mathrm{T}}=2.4 \mathrm{~T},<\mathrm{n}_{\mathrm{e}}>=3.810^{19} \mathrm{~m}^{-3}$, gas rate $\sim 1.8$ to $2.310^{22} \mathrm{Ds}^{-1}$ (injected at the top of the machine and at the divertor outer ring) and with $3.6 \mathrm{MW}$ of ICRH for auxiliary heating over a duration of $15 \mathrm{~s}$. 
This pulse is very comparable to those of the series except for the plasma geometry which exhibits different configurations during the pulse. Figure 4 displays the plasma

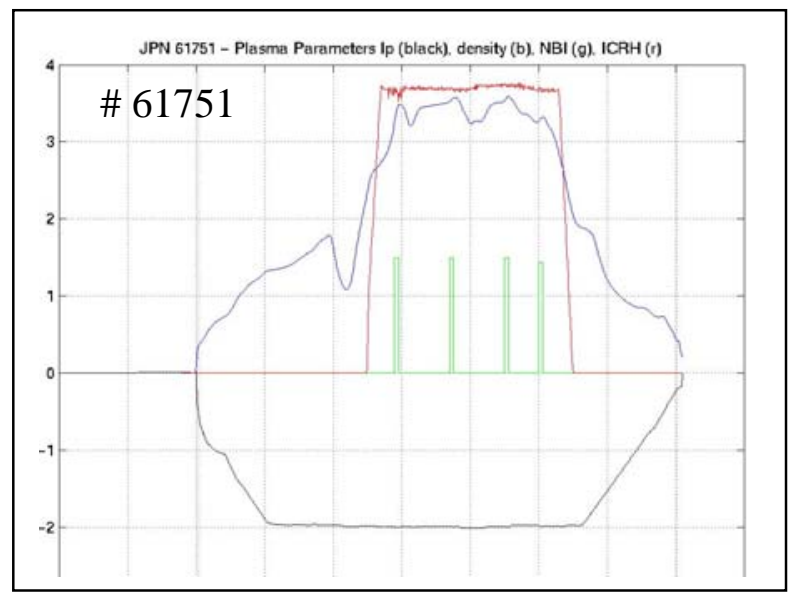

Figure 3: Plasma parameters as a function of time; the magnetic configuration is changed during the pulse geometry

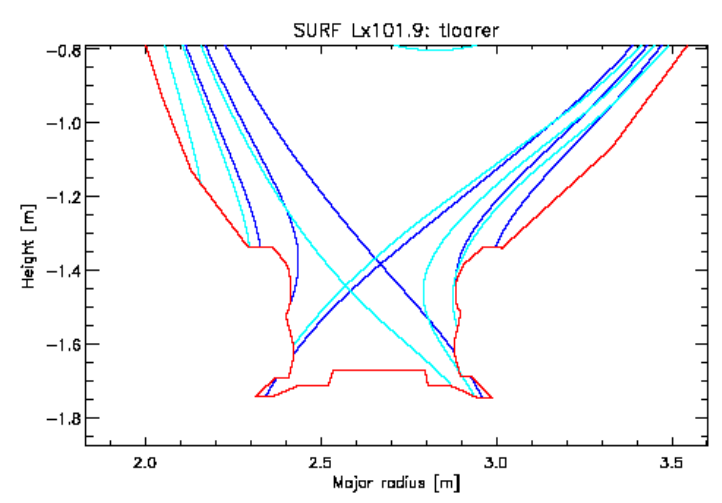

Figure 4 : Plasma geometry in the divertor at $t=58 \mathrm{sec}$ in dark (same geometry as for the series) and $t=62 \mathrm{sec}$ in liaht colours

divertor does not appear to be very sensitive to the outer strike point location, showing a rather weak dependence for the two phases and also during the transition phase (59 to $61 \mathrm{sec}$ ). The increase in the retention results from the higher gas injection necessary to maintain a constant plasma density. Finally, the particle exhaust increases regularly from $\sim 63$ to 65 sec, which is correlated with the final movement of the two strike points, returning to their initial location on the vertical targets. The corresponding wall loading also exhibits a decrease towards values close to those at the beginning of the pulse. geometry at 58 and $63 \mathrm{sec}$, while at the beginning of the pulse the two strike points were located on the horizontal target plates. Using the deduced pumping speed for the divertor pressure, the particle fluxes are displayed in figure 5 . The two phases considered can be seen in the time behaviour of the particle fluxes while the plasma density remains nearly constant over the pulse duration. In the first phase, [56-59], the plasma geometry is the same as for pulse 62925 while the outer strike point is located on the horizontal target in the phase [61-63], with $2 \mathrm{sec}$ of transition from one geometry to the other. In the first phase, the particle retention is about $25 \%$, while in the second phase it increases up to $40 \%$. It is worth noting that in this second phase the plasma geometry has a quasi double null configuration, increasing significantly the plasma interaction with the top of the machine and the associated particle retention. The neutral pressure in the

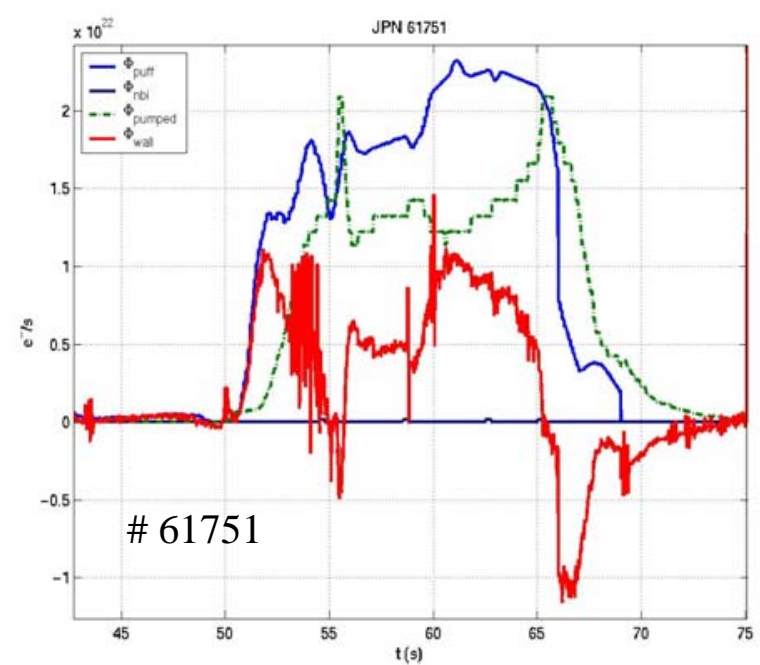

Figure 5: Time evolution of particle fluxes $\Gamma_{\text {puff, }} \Gamma_{\text {exhauted, }}$ and resulting wall particle retention $\Gamma_{\text {wall }}$. 


\section{IV - Particle recovery between pulses}

From this series of experiments, the particle recovery between pulses is shown to be rather weak in the global balance for the full day, showing that in previous results during the overall campaign this contribution was also overestimated [2]. However, it should be noted that the resulting neutral pressure in the vessel at the end of the discharge is not dependent of the retention observed during the pulse. The total retention at the end of each pulse of the series is always of the order of $1.010^{22} \mathrm{D}$, corresponding to $5 \%$ of the total injection. The quantity of gas released $100 \mathrm{sec}$ after the end of the pulse of the series is also very reproducible, at around $5.310^{21} \mathrm{D}$. For pulse \# 61751 (fig. 3), which exhibits a higher gas retention of $6.3610^{22} \mathrm{D}\left(22 \%\right.$ of the gas injected) "only" $4.4510^{21} \mathrm{D}$ are recovered $100 \mathrm{sec}$ after the end of the pulse. In spite of a higher gas retention, the corresponding particle recovery after this pulse is lower, showing that the gas released between pulses does not depend on the retention during the previous pulses or series of pulses. This behaviour has also been observed in Tore Supra by comparing the gas release for a full day of "short" pulse experiments ( 15-20 sec) with a full day of "long" discharges cumulating respectively in 546 and $1330 \mathrm{sec}$ of plasma [3]. The total gas released over the day for the two experiments is very similar in spite of a higher number of particles retained for the long discharges. This behaviour has been confirmed after a series of three consecutive discharges cumulating in more than 15 min of plasma duration with a constant gas retention of $\sim 210^{20} \mathrm{Ds}^{-1}$ over the plasma operation. The resulting gas released after each discharge is the same for each of these long discharges and "only" twice higher after these 6 min long discharges compared to the same pulse of $30 \mathrm{sec}$ duration [4].

\section{IV - Conclusions}

The assessment of the fuel retention over a full day of experiments in JET has been carried out to perform a gas balance with the maximum accuracy. The pumping speed of the cryopump in reference to the neutral pressure measurements has been estimated with and without plasma. Using these pumping speeds, the gas balance analysis over the full day of experiments in JET shows that the plasma geometry in the divertor influence the resulting particle retention, particularly for quasi double null configuration and that the gas released between pulses has been overestimated in previous JET gas balance analysis. This results in a lower retention during the plasma. However, this also leads to a lower recovery between pulses in addition to a weak dependence with the plasma conditions, duration and retention. This result is also observed on Tore Supra for long discharges showing a steady state retention but without any extra recovery after the pulse compared to short pulses.

\section{References}

[1] G. Federici, C. Skinner, J.N. Brooks et al., Nuclear Fusion, Vol.41, Vol.12R (2001).

[2] J Bucalossi et al., $28^{\text {th }}$ European Conference on Controlled Fusion and Plasma Physics - Funchal, 18-22 June 2001, ECA, Vol.25A (2001) 1629-1632.

[3] B. Pégourié et al., $10^{\text {th }}$ International Workshop on Carbon Materials for Fusion Application (2003), 17-19 September 2003, Juelich.

[4] A Grosman et al., to be published in Journal of Nucl. Material.

\section{Acknowledgements}

This work was performed under the European Fusion Development Agreement, and was partly funded by the UK Engineering and Physical Sciences Research Council and by EURATOM. 